\title{
ANÁLISE DO MERCADO INTERNACIONAL DE COMPENSADO ${ }^{1}$
}

\begin{abstract}
Antônio Donizette de Oliveira ${ }^{2}$, Ivonise Silva Andrade Ribeiro ${ }^{3}$ e José Roberto S. Scolforo ${ }^{2}$
RESUMO - Este estudo visou construir um modelo de comércio internacional de compensado para simular mudanças nos fluxos e preços deste produto, ocasionadas por alterações nos deslocadores da demanda dos principais países importadores e nos deslocadores da oferta dos principais exportadores. O modelo considera que as importações sejam diferenciadas pelo local de produção, significando que os produtos não são substitutos perfeitos. Os resultados indicaram que os aumentos da demanda de compensado no Japão, Estados Unidos e Alemanha tendem a elevar os preços dos países exportadores que têm participação maior naqueles mercados. Os países menos expressivos nos mercados em questão aumentam pouco ou até reduzem seus preços e, com isso, beneficiamse de incrementos maiores nos fluxos comerciais do que os países com participação maior. O crescimento da demanda de compensado do Japão afeta pouco os preços, os fluxos comerciais e a receita de exportação de compensado do Brasil. Já no caso do aumento das demandas da Alemanha e dos Estados Unidos há elevação dos preços e da receita de exportação brasileira, mas a quantidade total de compensado comercializado pelo país diminui. Um aumento exógeno na oferta de compensado de determinado país reduz seu preço, permitindo que haja uma ampliação de sua participação em todos os mercados. No caso do Brasil, o crescimento da oferta de compensado elevou a sua participação em todos os mercados, mas a receita total de exportação diminuiu, em decorrência da queda no preço do produto.
\end{abstract}

Palavras-chave: Compensado, comércio internacional, demanda e oferta.

\section{PLYWOOD INTERNATIONAL MARKET ANALYSIS}

\begin{abstract}
A plywood international market model considering the main importing and exporting countries was built. The world market was modeled to simulate changes in production, flow and prices caused by shocks in the exogenous variables such as supply and demand changes. The chosen model assumes that imports are differentiated by production sites, i.e., plywood products coming from different places are not perfect substitutes. The results suggest that a demand increase, in Japanese, North American and German plywood tend to increase the prices of export countries that have larger participation in those markets. The less expressive countries in plywood market, in turn, are subject to very small increase in their prices or even suffer a reduction in prices, thus benefitting more with the increases in the commercial flows than those countries with larger world market participation. The Japanese plywood demand growth has little effect on Brazilian plywood prices, commercial flows and export revenue. However, in the case of Germany and United States plywood demand increase there is a significant Brazilian price and export revenue increase, but the amount of plywood commercialized by the country diminishes. An exogenous increase in the amount of plywood produced by a country reduces its price, increasing its participation in all markets. In the Brazilian case, a plywood supply growth increased its participation in all markets, but the total gross export income decreased, due to the fall in the plywood world prices.
\end{abstract}

Key-words: Plywood, international trade, demand, supply

\footnotetext{
${ }^{1}$ Recebido em $1^{\circ} .09 .2003$ e aceito para publicação em 25.11.2004.

${ }^{2}$ Departamento de Ciências Florestais da UFLA-Cx.P.3037-37200-000 Lavras, MG. E-mail:<donizete@ufla.br>,<scolforo@ufla.br>.

${ }^{3}$ Programa de Pós-Graduação em Engenharia Florestal da UFLA - Cx.P. 3037 - 37200-000 Lavras - MG.
} 


\section{INTRODUÇÃO}

O compensado é um painel constituído de um conjunto de lâminas coladas com a grã alternada, geralmente em ângulo reto. As lâminas são, usualmente, coladas simetricamente a partir do centro ou miolo do painel, aos pares em ambos os lados. Sua produção em escala industrial iniciou em meados do século XX. Contudo, segundo Zugman (1994), os esquifes utilizados pelos egípcios há mais de 3.500 anos eram feitos de compensado, e no Império Romano a mobília era confeccionada usando compensado.

Em 2002, a produção mundial de compensado foi de 58,7 milhões de toneladas, concentrada em um pequeno número de países produtores, em que apenas os seis maiores respondem por $77 \%$ do volume total. Os Estados Unidos são os maiores produtores e respondem por $26,4 \%$ do total mundial. A seguir vêm a China, a Indonésia, a Malásia, o Brasil e o Japão (FAO, 2004).

O comércio internacional de compensado é significativo, tendo movimentado cerca de 6,54 bilhões de dólares em 2002. O principal mercado para esse produto é o asiático, cujas importações totalizaram 9,5 milhões de toneladas naquele ano, sendo mais de $72 \%$ comprados pelo Japão e pela China. O mercado norte-americano vem logo a seguir, com importações de 3,89 milhões de toneladas de compensado. Outro mercado importante é o europeu, onde países como Alemanha, Inglaterra, Bélgica, Itália e França participam ativamente desse comércio de compensado.

Em termos de exportação, Indonésia, Malásia, China, Brasil, Federação Russa, Finlândia, Canadá e Estados Unidos controlam mais de $82,6 \%$ do comércio internacional. Nos últimos anos, a Indonésia tem sido o maior exportador de compensado. Em 1990, suas exportações totalizaram 8,2 milhões de toneladas e representaram cerca de $52 \%$ do volume total de compensado transacionado no mercado internacional. Em 2002, suas vendas externas de compensado caíram para 5,5 milhões de toneladas, e sua participação no mercado recuou para 27,6\%. O Japão, a China e os Estados Unidos são os principais mercados para o compensado indonésio.

O Brasil ocupou, em 2002, a quarta posição no mundo entre os exportadores de compensado, tendo vendido cerca de 1,4 milhão de toneladas no mercado externo, gerando divisas da ordem de 387 milhões de dólares. Sua participação no mercado externo vem crescendo sistematicamente, tendo passado de $1,9 \%$ em 1990 para $7 \%$ em 2002. Os principais compradores do compensado brasileiro são os Estados Unidos e o Reino Unido.

O Brasil segue tendência oposta à mundial nas exportações de painéis de madeira. No caso do compensado, enquanto o mundo diminui as exportações, inversamente o Brasil as aumenta. Já com relação a painéis de partícula, painéis de fibra e laminados, enquanto o mundo aumenta suas exportações, o Brasil as diminui (BRASIL et al., 2003).

A tendência crescente das exportações brasileiras de painéis de madeira é dada pela perda de mercado interno do compensado para os painéis de partícula, painéis de fibra e laminados; assim, a alternativa para compensar as perdas com as quedas da demanda interna é encontrada nas exportações. Nesse contexto, o Brasil aproveita o mercado deixado pela Indonésia, Malásia e Estados Unidos para incrementar suas exportações (BRASIL, 2002; BRASIL et al., 2003).

Segundo Brasil et al. (2003), embora o compensado brasileiro tenha encontrado no curto prazo uma solução para a queda na demanda nacional, isso não significa que essa demanda esteja garantida no longo prazo, dada a tendência de queda na demanda mundial por esse produto.

Mudanças na estrutura de mercado e nas políticas de comércio exterior, juntamente com a evolução da capacidade produtiva dos países exportadores, podem alterar suas posições no mercado mundial de compensado. Umas das maneiras de prever essas mudanças é utilizar modelos econômicos que possibilitem analisar a dinâmica do mercado internacional de determinado produto, por meio da elaboração de cenários, que contemplem as alterações esperadas no comércio entre os diversos países, no curto e longo prazos.

Segundo Wack (1985) tem havido evolução relacionada com a utilização de modelos de planejamento estratégico, principalmente com a aplicação de técnicas de simulação para analisar futuros alternativos e diferentes estratégias de gestão. Essa abordagem incorpora a incerteza e é designada por análise de cenários, já que, em lugar de se prever o futuro, apresentam-se vários futuros plausíveis, dependentes de diferentes pressupostos. Algumas de suas aplicações recorrem à análise de sensibilidade, com um número limitado de elementos-chave, exógenos e endógenos. 
Nesse contexto, este estudo objetivou construir um modelo de comércio internacional de compensado para simular mudanças no curto prazo nos fluxos e nos preços deste produto, ocasionadas por choques em variáveis exógenas como mudanças nos deslocadores da demanda dos principais países importadores e nos deslocadores da oferta dos principais países exportadores.

\section{MATERIAL E MÉTODOS}

A formulação do modelo de comércio é baseada na construção de um sistema que representa o mercado mundial de compensado, constituída de equações de demanda, oferta e preços, como se segue:

Para cada país ou região importadora existe uma equação de demanda para cada produtor, dada por:

$$
D_{i j}=\eta_{i j j} P_{i j} \sum_{h=1}^{n} \eta_{i j h} P_{i h}+B_{i j}
$$

em que os índices i correspondem aos países importadores; os j, aos países exportadores; e os $\mathrm{h}$, aos compensados dos países competidores.

Existem, também, equações de oferta:

$$
Q_{j}=\varepsilon_{j} P_{j}+Z_{j}
$$

e equações que ligam os preços dos países importadores aos dos países exportadores:

$$
P_{i j}=t_{i j} P_{j}+T_{i j}
$$

O modelo completa-se com equações de identidade que mostram que as demandas de cada país individual e do Resto do Mundo (RM) devem igualar-se à oferta total de compensado do período:

$$
Q_{j}=\sum_{i=1}^{n} \alpha_{i j} D_{i j}+\beta_{r j} D_{r j}
$$

As variáveis do modelo podem ser definidas como:

$\mathrm{D}_{\mathrm{ij}}=$ mudança porcentual na quantidade de compensado do país j, que vai para o país i;

$\mathrm{P}_{\mathrm{ij}}=$ mudança porcentual no preço do compensado do país j, no país i;

$\mathrm{P}_{\mathrm{ih}}=$ mudança porcentual no preço do compensado do país h, no país i;

$\mathrm{B}_{\mathrm{ij}}=$ mudança porcentual na demanda correspondente a choques exógenos;

$\eta_{\mathrm{ijj}}=$ elasticidade-preço direta da demanda de compensado do país j, no país i; $\eta_{\mathrm{ijh}}=$ elasticidade-preço cruzada da demanda de compensado do país j, em relação ao preço do compensado do país h, no país i;

$\mathrm{P}_{\mathrm{j}}=$ mudança porcentual no preço do produtor de compensado, no país j;

$\mathrm{T}_{\mathrm{ij}}=$ mudança porcentual no diferencial de preços;

$\mathrm{Q}_{\mathrm{j}}=$ mudança porcentual na oferta de compensado, do país j;

$\varepsilon_{\mathrm{j}}=$ flexibilidade-preço da oferta do compensado, no país j;

$Z_{j}=$ mudança porcentual nos preços da oferta, correspondente a choques exógenos;

$\mathrm{t}_{\mathrm{ij}}=$ elasticidade de transmissão de preço do compensado do país j, no país i;

$\alpha_{\mathrm{ij}}$ e $\beta_{\mathrm{rj}}=$ proporção do compensado produzido no país j e exportado para o país i e para o resto do mundo $r$, respectivamente;

$D_{r j}=$ mudança porcentual na quantidade de celulose do país j, que vai para o resto do mundo.

O sistema envolve, potencialmente, $n^{2}$ equações de demanda e preço e $n$ equações de oferta e identidade, em que $n$ é o número de regiões endógenas do mercado. Contudo, o número empírico dessas equações é reduzido, visto que alguns fluxos de comércio não existem entre países, ou existem em quantidades negligenciáveis.

Para estudar o mercado internacional de compensado, foram selecionados, como países importadores, Reino Unido (RU), Alemanha (AL), Holanda (HO), Dinamarca (DI), França (FR), Bélgica (BE), Suíça (SU), Suécia (SE), Itália (IT), Estados Unidos (EU) e Japão (JA), além de uma região chamada de "Resto do Mundo" (R1), que engloba todos os demais países importadores. Como exportadores, foram selecionados Alemanha (AL), Áustria (AU), Bélgica (BE), Brasil (BR), Canadá (CA), Cingapura (CI), Estados Unidos (EU), Finlândia (FI), França (FR), Indonésia (IN), Itália (IT), Malásia (MA) e Suécia (SE). Os demais países exportadores foram agregados em uma região denominada "Resto do Mundo" (R2). A China, apesar de ter grande participação no mercado internacional como país exportador e importador de compensado, não foi incluída no modelo, por não se dispor de dados sobre os seus fluxos comerciais desse produto no formato requerido pelo modelo de comércio.

R. Árvore, Viçosa-MG, v.29, n.2, p.311-320, 2005 
O sistema apresenta, assim, 364 equações com 364 variáveis endógenas, 12 quantidades consumidas e 12 preços em cada uma das regiões importadoras, mais 14 quantidades ofertadas e 14 preços de oferta. As 364 equações formam uma matriz $\mathrm{X}=\mathrm{AY}$, em que X é um vetor 1 x 364 variáveis exógenas; A é a matriz 364 x 364 de parâmetros; e Y é um vetor 1x364 de variáveis endógenas. Por meio de $\mathrm{Y}=\mathrm{A}^{-1} \mathrm{X}$, obtêm-se as mudanças porcentuais nas variáveis endógenas resultantes de mudanças no vetor de variáveis exógenas.

As mudanças exógenas simuladas dizem respeito às mudanças nos deslocadores da demanda e da oferta de compensado. Essas alterações nos deslocadores exógenos da demanda podem ser consideradas como aumentos na renda e, ou, população nas regiões estudadas, enquanto as variações exógenas na oferta simularão os efeitos da produção no comércio de compensado.

Na solução de curto prazo, considera-se que a oferta é exógena ou fixa e, portanto, excluída do modelo. Assim, o sistema é constituído de equações de demanda, preço e identidade, conforme já especificadas. As elasticidades-preço diretas e cruzadas são elementos relacionados às equações de demanda. Neste trabalho foram usados os valores dessas elasticidades calculados por Ribeiro (2003). Os elementos necessários nas equações de preço são as elasticidades de transmissão de preços que indicam a variação porcentual nos preços domésticos, em dada variação porcentual nos preços do mercado internacional. Por serem as quantidades de compensado negociadas nos mercados internacionais expressas em dólares, a elasticidade-transmissão de preço é assumida como tendo o valor unitário (ALSTON, 1985; SILVA, 1990; OLIVEIRA, 1995), o que indica uma transmissão total dos preços internacionais nos preços domésticos. Os elementos das equações de identidade são as proporções da quantidade de compensado produzido no país j e exportado para o país i e para o resto do mundo, conforme apresentados no Quadro 1. Assim, por exemplo, os maiores compradores do compensado brasileiro são os países agregados na região denominada resto do mundo, com uma proporção de $24,03 \%$. A seguir vêm o Reino Unido e os Estados Unidos, com 22,67 e 22,22\%, respectivamente. Os demais importadores participam com porcentuais que variam de $0,053 \%$ para a Suíça a $12,58 \%$ para a Alemanha.
R. Árvore, Viçosa-MG, v.29, n.2, p.311-320, 2005
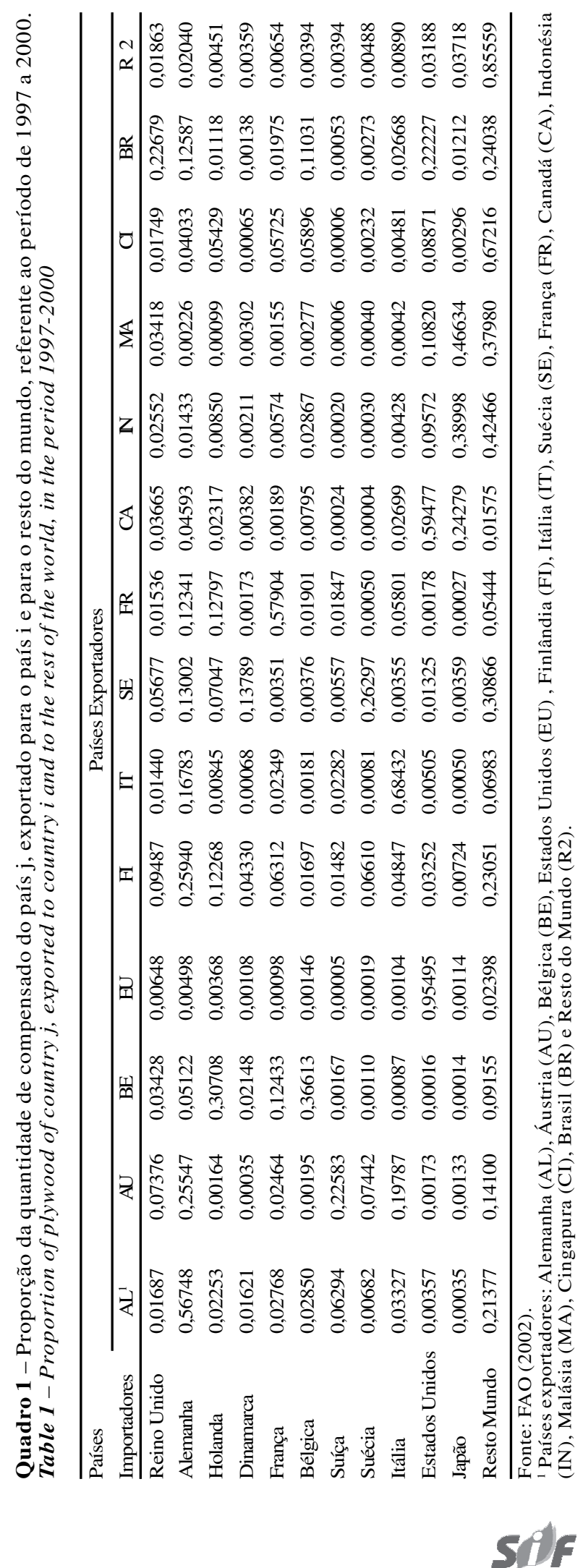


\section{RESULTADOS E DISCUSSÃO}

\subsection{Efeito de mudanças exógenas na demanda}

Nos Quadros 2, 3 e 4, mostram-se os efeitos nos fluxos e nos preços de deslocadores que aumentam em $10 \%$ a demanda de compensado do Japão, Estados Unidos e Alemanha, respectivamente. Os resultados das colunas 1 a 12 representam as mudanças porcentuais nos fluxos comerciais de compensado, enquanto a coluna 13 corresponde às mudanças nos preços desse produto. No caso de um aumento de $10 \%$ na demanda japonesa, Indonésia e Malásia seriam os maiores beneficiados em termos de elevação dos preços do compensado, com aumentos de 3,227 e 3,917\%, respectivamente. Em contrapartida, seus fluxos comerciais com aquele país aumentariam menos em termos porcentuais que os fluxos dos demais países exportadores. A grande participação da Indonésia (38,99\%) e da Malásia (46,63\%) no mercado japonês explicaria a elevação maior nos respectivos preços do compensado. O preço do compensado brasileiro aumentaria apenas $0,124 \%$, e suas exportações cairiam para Alemanha, EUA, Reino Unido, Holanda, França, Suíça, Suécia e Itália, mas elevariam para os outros países, principalmente o Japão $(7,785 \%)$.

O aumento de $10 \%$ na demanda dos Estados Unidos eleva o preço do compensado do Brasil $(2,47 \%)$, do Canadá $(7,39 \%)$, de Cingapura $(0,84 \%)$, dos Estados Unidos $(11,72 \%)$, da Indonésia $(0,79 \%)$, da Malásia $(0,88 \%)$ e do resto do mundo $(0,45 \%)$ e reduz o preço dos demais países exportadores. Para atender ao aumento de consumo de compensado do Brasil pelos Estados Unidos (7,307\%), o Brasil reduz suas vendas para todos os mercados, em porcentuais que variam de $1,11 \%$ para a Itália a $4,05 \%$ para a Suécia.

Com o aumento de $10 \%$ nos deslocadores da demanda alemã, os preços do compensado desse país subiram 7,50\%, o que reduziu as exportações da Alemanha para todos os mercados. Entretanto, o consumo de compensado doméstico cresceu 5,91\%. Todos os países aumentaram suas vendas para a Alemanha e, na maioria dos casos, reduziram as exportações para os outros mercados. No caso do Brasil, o aumento das exportações para a Alemanha foi de $8,582 \%$, mas houve redução das vendas para todos os demais países.

Quadro 2 - Efeito nas variáveis endógenas decorrentes de um aumento de 10\% nos deslocadores da demanda de compensado do Japão. Mudanças porcentuais de curto prazo

Table 2 - Effect of a 10\% increase in the demand shifters of Japanese plywood on endogenous variables. Short run per cent shifting

\begin{tabular}{lccccccccccccc}
\hline $\begin{array}{l}\text { Países } \\
\text { Exportadores }\end{array}$ & $\mathrm{D}_{\mathrm{ALj}}{ }^{1}$ & $\mathrm{D}_{\mathrm{EUj}}$ & $\mathrm{D}_{\mathrm{BEj}}$ & $\mathrm{D}_{\mathrm{RUj}}$ & $\mathrm{D}_{\mathrm{HOj}}$ & $\mathrm{D}_{\mathrm{DIj}}$ & $\mathrm{D}_{\mathrm{FRj}}$ & $\mathrm{D}_{\mathrm{Suj}}$ & $\mathrm{D}_{\mathrm{SEj}}$ & $\mathrm{D}_{\mathrm{ITj}}$ & $\mathrm{D}_{\mathrm{JAj}}$ & $\mathrm{D}_{\mathrm{Rlj}}$ & $\mathrm{P}_{\mathrm{j}}{ }^{2}$ \\
\hline Alemanha & $-0,263$ & $-0,023$ & 0,522 & $-0,425$ & $-0,420$ & 0,428 & 0,073 & $-0,314$ & $-0,425$ & 0,071 & 7,970 & 0,746 & $-0,130$ \\
Áustria & $-0,209$ & 0,067 & 0,631 & $-0,389$ & $-0,331$ & 0,548 & 0,157 & $-0,286$ & $-0,328$ & 0,128 & 8,060 & 0,923 & $-0,253$ \\
Bélgica & $-0,327$ & $-0,131$ & 0,391 & $-0,470$ & $-0,526$ & 0,283 & $-0,027$ & $-0,348$ & $-0,542$ & 0,003 & 7,862 & 0,533 & 0,018 \\
Brasil & $-0,373$ & $-0,208$ & 0,297 & $-0,501$ & $-0,603$ & 0,179 & $-0,099$ & $-0,372$ & $-0,625$ & $-0,046$ & 7,785 & 0,380 & 0,124 \\
Canadá & $-1,433$ & $-1,990$ & $-1,865$ & $-1,233$ & $-2,364$ & $-2,214$ & $-1,758$ & $-0,934$ & $-2,551$ & $-1,172$ & 6,003 & $-3,138$ & 2,568 \\
Cingapura & $-0,450$ & $-0,339$ & 0,138 & $-0,555$ & $-0,732$ & 0,003 & $-0,221$ & $-0,414$ & $-0,767$ & $-0,129$ & 7,654 & 0,122 & 0,303 \\
Estados Unidos & $-0,263$ & $-0,024$ & 0,521 & $-0,426$ & $-0,420$ & 0,427 & 0,073 & $-0,314$ & $-0,426$ & 0,071 & 7,969 & 0,745 & $-0,129$ \\
Finlândia & $-0,303$ & $-0,091$ & 0,439 & $-0,453$ & $-0,487$ & 0,336 & 0,010 & $-0,335$ & $-0,499$ & 0,028 & 7,902 & 0,612 & $-0,037$ \\
França & $-0,263$ & $-0,025$ & 0,520 & $-0,426$ & $-0,421$ & 0,426 & 0,072 & $-0,314$ & $-0,427$ & 0,070 & 7,968 & 0,743 & $-0,128$ \\
Indonésia & $-1,718$ & $-2,470$ & $-2,448$ & $-1,430$ & $-2,839$ & $-2,859$ & $-2,205$ & $-1,085$ & $-3,070$ & $-1,476$ & 5,522 & $-4,087$ & 3,227 \\
Itália & $-0,301$ & $-0,087$ & 0,444 & $-0,452$ & $-0,483$ & 0,342 & 0,014 & $-0,334$ & $-0,494$ & 0,031 & 7,906 & 0,619 & $-0,042$ \\
Malásia & $-2,017$ & $-2,973$ & $-3,059$ & $-1,637$ & $-3,336$ & $-3,535$ & $-2,674$ & $-1,244$ & $-3,613$ & $-1,794$ & 5,019 & $-5,080$ & 3,917 \\
Suécia & $-0,318$ & $-0,116$ & 0,409 & $-0,463$ & $-0,511$ & 0,303 & $-0,013$ & $-0,343$ & $-0,525$ & 0,013 & 7,877 & 0,563 & $-0,003$ \\
Resto Mundo & $-0,565$ & $-0,531$ & $-0,095$ & $-0,634$ & $-0,922$ & $-0,255$ & $-0,400$ & $-0,474$ & $-0,974$ & $-0,250$ & 7,462 & $-0,257$ & 0,567 \\
\hline
\end{tabular}

${ }^{1} \mathrm{D}_{\mathrm{ALj}} \mathrm{D}_{\mathrm{EUj}}, \mathrm{D}_{\mathrm{BEj}}, \mathrm{D}_{\mathrm{RUj}}, \mathrm{D}_{\mathrm{HO}}, \mathrm{D}_{\mathrm{DIj}}, \mathrm{D}_{\mathrm{FRj}}, \mathrm{D}_{\mathrm{SUj}}, \mathrm{D}_{\mathrm{SEj}}, \mathrm{D}_{\mathrm{ITj}}, \mathrm{D}_{\mathrm{JAj}}$ e $\mathrm{D}_{\mathrm{RIj}}$ são as mudanças porcentuais nos fluxos de comércio do compensado do país j, na Alemanha, Estados Unidos, Bélgica, Reino Unido, Holanda, Dinamarca, França, Suíça, Suécia, Itália, Japão e no Resto do Mundo, respectivamente.

${ }^{2}$ As mudanças nos preços $\left(\mathrm{P}_{\mathrm{j}}\right)$ são as mesmas em todos os mercados, para o compensado oriundo dos países exportadores e do Resto do Mundo. 
Quadro 3 - Efeito nas variáveis endógenas decorrentes de um aumento de 10\% nos deslocadores da demanda de compensado dos Estados Unidos. Mudanças porcentuais de curto prazo

Table 3 - Effect of a $10 \%$ increase in the demand shifters of United States plywood on endogenous variables. Short run per cent shifting

\begin{tabular}{lccccccccccccc}
\hline $\begin{array}{l}\text { Países } \\
\text { Exportadores }\end{array}$ & $\mathrm{D}_{\mathrm{ALj}}{ }^{1}$ & $\mathrm{D}_{\mathrm{EUj}}$ & $\mathrm{D}_{\mathrm{BEj}}$ & $\mathrm{D}_{\mathrm{RUj}}$ & $\mathrm{D}_{\mathrm{HOj}}$ & $\mathrm{D}_{\mathrm{DIj}}$ & $\mathrm{D}_{\mathrm{FRj}}$ & $\mathrm{D}_{\mathrm{Suj}}$ & $\mathrm{D}_{\mathrm{SEj}}$ & $\mathrm{D}_{\mathrm{ITj}}$ & $\mathrm{D}_{\mathrm{JAj}}$ & $\mathrm{D}_{\mathrm{Rlj}}$ & $\mathrm{P}_{\mathrm{j}}{ }^{2}$ \\
\hline Alemanha & $-0,467$ & 9,471 & 0,608 & $-0,402$ & $-0,605$ & 1,418 & 0,318 & $-0,633$ & $-1,712$ & 0,255 & $-0,197$ & 1,148 & $-0,503$ \\
Áustria & $-0,326$ & 9,708 & 0,896 & $-0,305$ & $-0,370$ & 1,737 & 0,539 & $-0,558$ & $-1,456$ & 0,405 & 0,040 & 1,617 & $-0,829$ \\
Bélgica & $-0,559$ & 9,316 & 0,421 & $-0,466$ & $-0,757$ & 1,211 & 0,174 & $-0,682$ & $-1,879$ & 0,158 & $-0,352$ & 0,843 & $-0,291$ \\
Brasil & $-1,754$ & 7,307 & $-2,019$ & $-1,291$ & $-2,744$ & $-1,489$ & $-1,697$ & $-1,315$ & $-4,051$ & $-1,113$ & $-2,362$ & $-3,126$ & 2,466 \\
Canadá & $-3,891$ & 3,715 & $-6,379$ & $-2,767$ & $-6,296$ & $-6,314$ & $-5,042$ & $-2,447$ & $-7,933$ & $-3,383$ & $-5,955$ & $-10,219$ & 7,393 \\
Cingapura & $-1,051$ & 8,490 & $-0,583$ & $-0,805$ & $-1,575$ & 0,100 & $-0,596$ & $-0,942$ & $-2,773$ & $-0,365$ & $-1,179$ & $-0,790$ & 0,843 \\
Estados Unidos & $-5,767$ & 0,562 & $-10,206$ & $-4,062$ & $-9,413$ & $-10,549$ & $-7,977$ & $-3,441$ & $-11,340$ & $-5,377$ & $-9,109$ & $-16,44611,718$ \\
Finlândia & $-0,647$ & 9,168 & 0,240 & $-0,527$ & $-0,904$ & 1,011 & 0,036 & $-0,728$ & $-2,040$ & 0,064 & $-0,500$ & 0,550 & $-0,087$ \\
França & $-0,556$ & 9,321 & 0,426 & $-0,464$ & $-0,753$ & 1,217 & 0,178 & $-0,680$ & $-1,874$ & 0,161 & $-0,347$ & 0,852 & $-0,297$ \\
Indonésia & $-1,028$ & 8,528 & $-0,536$ & $-0,790$ & $-1,537$ & 0,152 & $-0,560$ & $-0,930$ & $-2,731$ & $-0,340$ & $-1,140$ & $-0,713$ & 0,790 \\
Itália & $-0,637$ & 9,185 & 0,261 & $-0,520$ & $-0,888$ & 1,033 & 0,051 & $-0,723$ & $-2,022$ & 0,074 & $-0,484$ & 0,583 & $-0,110$ \\
Malásia & $-1,066$ & 8,465 & $-0,613$ & $-0,816$ & $-1,600$ & 0,066 & $-0,619$ & $-0,950$ & $-2,800$ & $-0,381$ & $-1,204$ & $-0,839$ & 0,877 \\
Suécia & $-0,513$ & 9,393 & 0,514 & $-0,434$ & $-0,681$ & 1,314 & 0,246 & $-0,657$ & $-1,796$ & 0,206 & $-0,275$ & 0,995 & $-0,397$ \\
Resto Mundo & $-0,880$ & 8,776 & $-0,235$ & $-0,688$ & $-1,292$ & 0,485 & $-0,329$ & $-0,852$ & $-2,463$ & $-0,184$ & $-0,892$ & $-0,224$ & 0,450 \\
\hline
\end{tabular}

Quadro 4 - Efeito nas variáveis endógenas decorrentes de um aumento de $10 \%$ nos deslocadores da demanda de compensado da Alemanha. Mudanças porcentuais de curto prazo

Table 4 -Effect of a 10\% increase in the demand shifters of German plywood on endogenous variables. Short run percent shifting

\begin{tabular}{lcccccccccccccc}
\hline $\begin{array}{l}\text { Países } \\
\text { Exportadores }\end{array}$ & $\mathrm{D}_{\mathrm{ALj}}{ }^{1}$ & $\mathrm{D}_{\mathrm{EUj}}$ & $\mathrm{D}_{\mathrm{BEj}}$ & $\mathrm{D}_{\mathrm{RUj}}$ & $\mathrm{D}_{\mathrm{HOj}}$ & $\mathrm{D}_{\mathrm{Dlj}}$ & $\mathrm{D}_{\mathrm{FRj}}$ & $\mathrm{D}_{\mathrm{Suj}}$ & $\mathrm{D}_{\mathrm{SEj}}$ & $\mathrm{D}_{\mathrm{ITj}}$ & $\mathrm{D}_{\mathrm{JAj}}$ & $\mathrm{D}_{\mathrm{Rij}}$ & $\mathrm{P}_{\mathrm{j}}{ }^{2}$ \\
\hline Alemanha & 5,912 & $-5,996$ & $-6,442$ & $-2,642$ & $-5,939$ & $-7,196$ & $-5,168$ & $-5,218$ & $-5,983$ & $-3,437$ & $-6,455$ & $-10,413$ & 7,502 \\
Áustria & 7,948 & $-2,574$ & $-2,288$ & $-1,237$ & $-2,556$ & $-2,599$ & $-1,981$ & $-4,139$ & $-2,285$ & $-1,274$ & $-3,032$ & $-3,655$ & 2,808 \\
Bélgica & 8,959 & $-0,873$ & $-0,223$ & $-0,538$ & $-0,874$ & $-0,315$ & $-0,398$ & $-3,603$ & $-0,447$ & $-0,199$ & $-1,331$ & $-0,295$ & 0,475 \\
Brasil & 8,582 & $-1,507$ & $-0,993$ & $-0,799$ & $-1,501$ & $-1,166$ & $-0,988$ & $-3,803$ & $-1,132$ & $-0,600$ & $-1,965$ & $-1,547$ & 1,345 \\
Canadá & 9,258 & $-0,371$ & 0,385 & $-0,332$ & $-0,378$ & 0,359 & 0,069 & $-3,445$ & 0,095 & 0,118 & $-0,829$ & 0,695 & $-0,213$ \\
Cingapura & 8,964 & $-0,864$ & $-0,213$ & $-0,535$ & $-0,866$ & $-0,303$ & $-0,390$ & $-3,600$ & $-0,437$ & $-0,194$ & $-1,322$ & $-0,279$ & 0,464 \\
Estados Unidos & 9,430 & $-0,081$ & 0,738 & $-0,213$ & $-0,091$ & 0,749 & 0,339 & $-3,353$ & 0,409 & 0,302 & $-0,539$ & 1,268 & $-0,611$ \\
Finlândia & 7,873 & $-2,699$ & $-2,440$ & $-1,288$ & $-2,680$ & $-2,768$ & $-2,098$ & $-4,179$ & $-2,420$ & $-1,353$ & $-3,158$ & $-3,902$ & 2,980 \\
França & 8,538 & $-1,581$ & $-1,083$ & $-0,829$ & $-1,574$ & $-1,266$ & $-1,057$ & $-3,826$ & $-1,212$ & $-0,647$ & $-2,040$ & $-1,694$ & 1,447 \\
Indonésia & 9,228 & $-0,421$ & 0,325 & $-0,353$ & $-0,427$ & 0,292 & 0,023 & $-3,460$ & 0,042 & 0,087 & $-0,879$ & 0,597 & $-0,145$ \\
Itália & 7,944 & $-2,579$ & $-2,295$ & $-1,239$ & $-2,561$ & $-2,607$ & $-1,987$ & $-4,141$ & $-2,291$ & $-1,278$ & $-3,038$ & $-3,665$ & 2,816 \\
Malásia & 9,325 & $-0,259$ & 0,522 & $-0,286$ & $-0,267$ & 0,510 & 0,174 & $-3,409$ & 0,217 & 0,189 & $-0,717$ & 0,917 & $-0,367$ \\
Suécia & 8,596 & $-1,484$ & $-0,966$ & $-0,789$ & $-1,478$ & $-1,136$ & $-0,967$ & $-3,796$ & $-1,107$ & $-0,585$ & $-1,942$ & $-1,503$ & 1,314 \\
Resto Mundo & 9,020 & $-0,771$ & $-0,100$ & $-0,496$ & $-0,773$ & $-0,178$ & $-0,303$ & $-3,571$ & $-0,337$ & $-0,135$ & $-1,229$ & $-0,095$ & 0,336 \\
\hline
\end{tabular}

\subsection{Efeito de mudanças exógenas na oferta}

O efeito de mudanças na produção, nos fluxos e no preço do compensado ofertado pelos diversos países foram simulados no modelo por meio de alterações nas equações de identidade, o que refletiu mudanças na oferta no curto prazo.

Os resultados do aumento de $10 \%$ na produção da Indonésia, Malásia e Brasil estão nos Quadros 5,
6 e 7, respectivamente. O aumento de $10 \%$ na oferta de compensado da Indonésia fez o seu preço de exportação cair $8,09 \%$ e o consumo de compensado indonésio aumentar em todos os países importadores, sendo esse aumento de $1,79 \%$ na Suíça a $11,05 \%$ no Japão. A Malásia aumentou em 3,52\% suas exportações para o Japão, mas perdeu mercado nos demais países importadores, em decorrência da elevação de 2,24\% no preço da exportação de seu compensado.

R. Árvore, Viçosa-MG, v.29, n.2, p.311-320, 2005 
Quadro 5 - Efeito nas variáveis endógenas decorrentes de um aumento de $10 \%$ nos deslocadores da oferta de compensado da Indonésia. Mudanças porcentuais de curto prazo

Table 5 - Effect of a $10 \%$ increase in the demand shifters of Indonesian plywood on endogenous variables. Short run per cent shifting

\begin{tabular}{|c|c|c|c|c|c|c|c|c|c|c|c|c|c|}
\hline $\begin{array}{l}\text { Países } \\
\text { Exportadores } \\
\end{array}$ & $\mathrm{D}_{\mathrm{ALj} j}{ }^{1}$ & $\mathrm{D}_{\mathrm{EUj}}$ & $\mathrm{D}_{\mathrm{BEj}}$ & $\mathrm{D}_{\mathrm{RUj}}$ & $\mathrm{D}_{\mathrm{HOj}}$ & $\mathrm{D}_{\mathrm{DIj}}$ & $\mathrm{D}_{\mathrm{FRj}}$ & $\mathrm{D}_{\text {Suj }}$ & $\mathrm{D}_{\mathrm{SEj}}$ & $\mathrm{D}_{\mathrm{ITj}}$ & $\mathrm{D}_{\mathrm{JAj}}$ & $\mathrm{D}_{\mathrm{Rlj}}$ & $\overline{\mathrm{P}_{j}^{2}}$ \\
\hline$\overline{\text { Ale }}$ & & & & 3 & & & & & & & 50 & & \\
\hline & & & & & & & & & & & & & \\
\hline & & & & 732 & & & & & & & & & J, \\
\hline & & & & & & & & & & & & & 0,01 \\
\hline & & & & & & & & & & & & & 1,929 \\
\hline & & & & & & & & & & & & & $-0,339$ \\
\hline & & & & & & & & & & & & & 0,19 \\
\hline & & & & & & & & & & & & & $-0,023$ \\
\hline & & & & & & & & & & & & & 0,011 \\
\hline & & & & & & & & & & & & & $-8,086$ \\
\hline & & & & & & & & & & & & & $-0,040$ \\
\hline & & & & & & & & & & & & & 2,24 \\
\hline & & & & & & & & & & & & & 10 \\
\hline Resto $\mathrm{I}$ & 0,197 & 331 & $-0,660$ & 0,705 & 0,424 & 0,198 & 0,169 & $-0,018$ & $-0,073$ & 0,102 & 5,326 & $-0,266$ & $-0,23$ \\
\hline
\end{tabular}

${ }^{1} \mathrm{D}_{\mathrm{ALj}} \mathrm{D}_{\mathrm{EUj}}, \mathrm{D}_{\mathrm{BEj}}, \mathrm{D}_{\mathrm{RUj}}, \mathrm{D}_{\mathrm{HO}}, \mathrm{D}_{\mathrm{DIj}}, \mathrm{D}_{\mathrm{FRj}}, \mathrm{D}_{\mathrm{SUj}}, \mathrm{D}_{\mathrm{SEj}}, \mathrm{D}_{\mathrm{ITj}}, \mathrm{D}_{\mathrm{JAj}}$ e $\mathrm{D}_{\mathrm{R} I \mathrm{j}}$ são as mudanças porcentuais nos fluxos de comércio do compensado do país j, na Alemajnha, Estados Unidos, Bélgica, Reino Unido, Holanda, Dinamarca, França, Suíça, Suécia, Itália, Japão e no Resto do Mundo, respectivamente.

${ }^{2}$ As mudanças nos preços $\left(\mathrm{P}_{\mathrm{j}}\right)$ são as mesmas em todos os mercados, para o compensado oriundo dos países exportadores e do Resto do Mundo.

Quadro 6 - Efeito nas variáveis endógenas decorrentes de um aumento de $10 \%$ nos deslocadores da oferta de compensado da Malásia. Mudanças porcentuais de curto prazo

Table 6 - Effect of a 10\% increase in the demand shifters of Malaysian plywood on endogenous variables. Short run per cent shifting

\begin{tabular}{lccccccccccccc}
\hline $\begin{array}{l}\text { Países } \\
\text { Exportadores }\end{array}$ & $\mathrm{D}_{\mathrm{ALj}}{ }^{1}$ & $\mathrm{D}_{\mathrm{EUj}}$ & $\mathrm{D}_{\mathrm{BEj}}$ & $\mathrm{D}_{\mathrm{RUj}}$ & $\mathrm{D}_{\mathrm{HOj}}$ & $\mathrm{D}_{\mathrm{DIj}}$ & $\mathrm{D}_{\mathrm{FRj}}$ & $\mathrm{D}_{\mathrm{Suj}}$ & $\mathrm{D}_{\mathrm{SEj}}$ & $\mathrm{D}_{\mathrm{ITj}}$ & $\mathrm{D}_{\mathrm{JAj}}$ & $\mathrm{D}_{\mathrm{R} 1 \mathrm{j}}$ & $\mathrm{P}_{\mathrm{j}}{ }^{2}$ \\
\hline Alemanha & 0,0194 & 0,0101 & $-0,0411$ & 0,0160 & 0,0901 & $-0,0010$ & 0,0043 & 0,0083 & 0,0156 & 0,0002 & 0,0207 & $-0,0603$ & $-0,0019$ \\
Áustria & 0,0186 & 0,0089 & $-0,0426$ & 0,0155 & 0,0890 & $-0,0026$ & 0,0032 & 0,0079 & 0,0143 & $-0,0005$ & 0,0195 & $-0,0627$ & $-0,0003$ \\
Bélgica & 0,0145 & 0,0019 & $-0,0511$ & 0,0126 & 0,0820 & $-0,0120$ & $-0,0033$ & 0,0057 & 0,0068 & $-0,0050$ & 0,0125 & $-0,0765$ & 0,0093 \\
Brasil & 0,0246 & 0,0189 & $-0,0305$ & 0,0196 & 0,0988 & 0,0108 & 0,0125 & 0,0111 & 0,0251 & 0,0058 & 0,0295 & $-0,0430$ & $-0,0140$ \\
Canadá & 0,0112 & $-0,0037$ & $-0,0579$ & 0,0103 & 0,0765 & $-0,0195$ & $-0,0085$ & 0,0040 & 0,0007 & $-0,0085$ & 0,0070 & $-0,0875$ & 0,0170 \\
Cingapura & 0,0324 & 0,0320 & $-0,0146$ & 0,0250 & 0,1118 & 0,0284 & 0,0247 & 0,0152 & 0,0393 & 0,0140 & 0,0426 & $-0,0171$ & $-0,0319$ \\
Estados Unidos & 0,0143 & 0,0016 & $-0,0515$ & 0,0125 & 0,0817 & $-0,0125$ & $-0,0036$ & 0,0056 & 0,0064 & $-0,0052$ & 0,0122 & $-0,0772$ & 0,0098 \\
Finlândia & 0,0166 & 0,0055 & $-0,0467$ & 0,0141 & 0,0856 & $-0,0072$ & 0,0000 & 0,0069 & 0,0106 & $-0,0027$ & 0,0161 & $-0,0694$ & 0,0044 \\
França & 0,0111 & $-0,0038$ & $-0,0580$ & 0,0103 & 0,0764 & $-0,0196$ & $-0,0086$ & 0,0039 & 0,0006 & $-0,0085$ & 0,0069 & $-0,0877$ & 0,0171 \\
Indonésia & 0,0263 & 0,0217 & $-0,0270$ & 0,0208 & 0,1016 & 0,0146 & 0,0151 & 0,0120 & 0,0282 & 0,0076 & 0,0324 & $-0,0373$ & $-0,0179$ \\
Itália & 0,0190 & 0,0094 & $-0,0419$ & 0,0157 & 0,0895 & $-0,0019$ & 0,0037 & 0,0081 & 0,0149 & $-0,0002$ & 0,0201 & $-0,0616$ & $-0,0010$ \\
Malásia & 4,4289 & 7,4221 & 8,9563 & 3,0604 & 7,4187 & 9,9551 & 6,9057 & 2,3446 & 8,0260 & 4,6858 & 7,4350 & $14,5779-10,1694$ \\
Suécia & 0,0216 & 0,0138 & $-0,0366$ & 0,0175 & 0,0938 & 0,0040 & 0,0078 & 0,0095 & 0,0196 & 0,0026 & 0,0245 & $-0,0530$ & $-0,0070$ \\
Resto Mundo & 0,0356 & 0,0374 & $-0,0080$ & 0,0272 & 0,1171 & 0,0357 & 0,0297 & 0,0169 & 0,0451 & 0,0175 & 0,0480 & $-0,0064$ & $-0,0394$ \\
\hline
\end{tabular}

O Japão aumentou sua compra de compensado de todos os fornecedores. Com isso, países como o Brasil, reduziram seus fluxos comerciais com a Dinamarca $(0,05 \%)$, Bélgica $(0,89 \%)$, Itália $(0,015 \%)$, França $(0,003 \%)$, Suíça $(0,08 \%)$, Suécia $(0,273 \%)$ e resto do mundo $(0,63 \%)$ para ofertarem mais compensado ao Japão.
No Quadro 6, mostra-se que um aumento de $10 \%$ na oferta do compensado da Malásia reduziria seu preço em $10,17 \%$ e aumentaria a demanda do compensado malaio em todos os mercados. Os preços dos demais exportadores, exceto Bélgica, Canadá, Estados Unidos, Finlândia e França, diminuíram em porcentuais baixos. 
Aumento de $10 \%$ na oferta de compensado brasileiro reduziria o preço desse produto no mercado internacional em $11,73 \%$ no Brasil, $0,20 \%$ na Suécia, $0,05 \%$ na França, $0,16 \%$ na Alemanha, $0,31 \%$ na Áustria, $0,04 \%$ na Bélgica e $0,02 \%$ na Itália. Já nos demais exportadores haveria aumento no preço do compensado. O baixo preço do compensado brasileiro faria com que os países importadores reduzissem suas compras de alguns fornecedores e as direcionassem principalmente para o Brasil, ou seja, aumentariam os fluxos do compensado brasileiro para todos os países importadores.

\subsection{Efeito absoluto das simulações}

Os resultados já apresentados evidenciaram as mudanças porcentuais nos fluxos e preços do compensado, em relação às simulações feitas no modelo de comércio, mas não indicaram a magnitude daquelas mudanças, uma vez que os países têm participações diferentes no mercado internacional de compensado. Nessa seção, analisaram-se os efeitos absolutos das simulações no Brasil, cujos resultados se encontram no Quadro 8.

Para calcular as mudanças absolutas nos fluxos e preços do compensado, dada qualquer alteração nas variáveis exógenas do sistema, tomaram-se como base os valores dos preços de exportação e dos fluxos comerciais referentes ao ano 2000 . Notou-se que naquele ano as exportações brasileiras de compensado atingiram
1,38 milhão de toneladas e geraram uma receita total de 373,6 milhões de dólares. O preço médio de venda do compensado foi de US\$270,00/tonelada.

Um aumento de $10 \%$ na demanda de compensado do Japão teve pouco efeito no preço (+ US\$0,33), na quantidade ( +6 toneladas) e na receita estimada das exportações (+US\$458.342,00). Com o aumento na demanda dos Estados Unidos, as exportações brasileiras caíram em todos os países, exceto nos Estados Unidos, onde aumentaram para 278.236 toneladas. Houve aumento do preço (+US\$ 6,66/tonelada), mas o total das exportações caiu em 4.605 toneladas. Apesar disso, a receita total das exportações brasileiras cresceram em 7,94 milhões de dólares.

Os aumentos na oferta de compensado da Indonésia e da Malásia tiveram pouco efeito absoluto sobre os fluxos, os preços, a quantidade total exportada e a receita das exportações brasileiras. No entanto, o aumento na oferta de compensado brasileiro implicou efeitos maiores em seus fluxos comerciais com os países importadores. Por exemplo, as exportações para os Estados Unidos tiveram acréscimo de mais de $32 \mathrm{mil}$ toneladas. O preço do compensado brasileiro caiu US\$ $31,69 /$ tonelada, enquanto a quantidade total de compensado exportado aumentou 141 mil toneladas. O resultado final foi uma queda na receita das exportações de mais de 10 milhões de dólares.

Quadro 7 - Efeito nas variáveis endógenas decorrentes de um aumento de 10\% nos deslocadores da oferta de compensado do Brasil. Mudanças porcentuais de curto prazo

Table 7 - Effect of a 10\% increase in the demand shifters of Brazilian plywood on endogenous variables. Short run percent shifting

\begin{tabular}{lccccccccccccc}
\hline $\begin{array}{l}\text { Países } \\
\text { Exportadores }\end{array}$ & $\mathrm{D}_{\mathrm{ALj}}{ }^{1}$ & $\mathrm{D}_{\mathrm{EUj}}$ & $\mathrm{D}_{\mathrm{BEj}}$ & $\mathrm{D}_{\mathrm{RUj}}$ & $\mathrm{D}_{\mathrm{HOj}}$ & $\mathrm{D}_{\mathrm{DIj}}$ & $\mathrm{D}_{\mathrm{FRj}}$ & $\mathrm{D}_{\mathrm{Suj}}$ & $\mathrm{D}_{\mathrm{SEj}}$ & $\mathrm{D}_{\mathrm{ITj}}$ & $\mathrm{D}_{\mathrm{JAj}}$ & $\mathrm{D}_{\mathrm{R} 1 \mathrm{j}}$ & $\mathrm{P}_{\mathrm{j}}{ }^{2}$ \\
\hline Alemanha & $-0,081$ & 4,243 & 0,127 & 0,193 & 0,037 & 0,412 & 0,103 & $-0,309$ & $-0,766$ & 0,080 & $-0,031$ & 0,167 & $-0,160$ \\
Áustria & $-0,017$ & 4,352 & 0,259 & 0,238 & 0,145 & 0,559 & 0,204 & $-0,274$ & $-0,648$ & 0,149 & 0,078 & 0,382 & $-0,309$ \\
Bélgica & $-0,133$ & 4,157 & 0,022 & 0,158 & $-0,048$ & 0,296 & 0,022 & $-0,336$ & $-0,859$ & 0,025 & $-0,118$ & $-0,004$ & $-0,042$ \\
Brasil & 4,940 & 12,683 & 10,373 & 3,660 & 8,382 & 11,749 & 7,961 & 2,352 & 8,355 & 5,415 & 8,411 & $16,836-11,738$ \\
Canadá & $-1,635$ & 1,631 & $-3,043$ & $-0,879$ & $-2,545$ & $-3,096$ & $-2,329$ & $-1,132$ & $-3,589$ & $-1,571$ & $-2,644$ & $-4,991$ & 3,423 \\
Cingapura & $-0,264$ & 3,935 & $-0,246$ & 0,067 & $-0,267$ & $-0,001$ & $-0,184$ & $-0,406$ & $-1,099$ & $-0,115$ & $-0,339$ & $-0,440$ & 0,262 \\
Estados Unidos & $-2,453$ & 0,256 & $-4,712$ & $-1,444$ & $-3,905$ & $-4,943$ & $-3,609$ & $-1,565$ & $-5,075$ & $-2,440$ & $-4,019$ & $-7,707$ & 5,309 \\
Finlândia & $-0,168$ & 4,098 & $-0,049$ & 0,134 & $-0,106$ & 0,217 & $-0,033$ & $-0,355$ & $-0,923$ & $-0,012$ & $-0,177$ & $-0,120$ & 0,039 \\
França & $-0,130$ & 4,161 & 0,028 & 0,160 & $-0,044$ & 0,303 & 0,027 & $-0,334$ & $-0,854$ & 0,028 & $-0,113$ & 0,006 & $-0,048$ \\
Indonésia & $-0,283$ & 3,904 & $-0,284$ & 0,054 & $-0,298$ & $-0,043$ & $-0,213$ & $-0,416$ & $-1,133$ & $-0,134$ & $-0,370$ & $-0,503$ & 0,305 \\
Itália & $-0,141$ & 4,143 & 0,006 & 0,152 & $-0,062$ & 0,278 & 0,009 & $-0,340$ & $-0,874$ & 0,017 & $-0,131$ & $-0,031$ & $-0,023$ \\
Malásia & $-0,309$ & 3,861 & $-0,336$ & 0,037 & $-0,340$ & $-0,101$ & $-0,253$ & $-0,429$ & $-1,179$ & $-0,162$ & $-0,413$ & $-0,587$ & 0,364 \\
Suécia & $-0,065$ & 4,270 & 0,160 & 0,205 & 0,064 & 0,449 & 0,128 & $-0,300$ & $-0,737$ & 0,097 & $-0,004$ & 0,220 & $-0,197$ \\
Resto Mundo & $-0,173$ & 4,090 & $-0,059$ & 0,130 & $-0,114$ & 0,206 & $-0,040$ & $-0,357$ & $-0,932$ & $-0,017$ & $-0,185$ & $-0,136$ & 0,050 \\
\hline
\end{tabular}

R. Árvore, Viçosa-MG, v.29, n.2, p.311-320, 2005 


\section{CONCLUSÕES}

Com base nos resultados, pode-se concluir que:

- Os aumentos da demanda de compensado no Japão, Estados Unidos e Alemanha tendem a aumentar os preços dos países exportadores que têm participação maior naqueles mercados. Os países menos expressivos nos mercados em questão aumentam pouco, ou até reduzem, seus preços e, com isso, beneficiam-se de aumentos maiores nos fluxos comerciais do que os países com participação maior.

- O crescimento da demanda de compensado do Japão afeta pouco os preços, os fluxos comerciais e a receita de exportação de compensado do Brasil. Já no caso do aumento das demandas da Alemanha e Estados Unidos há elevação dos preços e da receita de exportação brasileira, mas a quantidade total de compensado comercializado pelo país diminui.

- Um aumento exógeno na oferta de compensado de determinado país reduz seu preço, permitindo que haja ampliação de sua participação em todos os mercados. No caso do Brasil, o crescimento da oferta de compensado elevou sua participação em todos os mercados, mas a receita total de exportação diminuiu, em decorrência da queda no preço do produto.

\section{REFERÊNCIAS BIBLIOGRÁFICAS}

\section{Alston, J. M. The effect of the}

European community's common agticultural policy in international markets for poutry meat. Raleigh: North Carolina Agricultural Research Service, 1985. 181p. (Bulletin n. 71).

BRASIL, A.A. et al. Demanda de exportação de painéis de madeira do Brasil. Floresta, v. 33, n.2, p. 135-146, 2003.

BRASIL, A.A. As exportações brasileiras de painéis de madeira. 2002. 74f. Dissertação (Mestrado em Ciências Florestais) Universidade Federal do Paraná, Curitiba, 2002.

FAO. Base de dados FAOSTAT.

Disponível em: <http:/apps.fao.org> Acesso em: 17 nov. 2002.

R. Árvore, Viçosa-MG, v.29, n.2, p.311-320, 2005 
FAO. Base de dados FAOSTAT.

Disponível em: <http:/apps.fao.org> Acesso em: 10 set. 2004.

FAO. FAO Provisional Outlook for Global Forest Products Consumption, Production and Trade to 2010. Forest Policy and Planning

Division. Rome: 1997.

OLIVEIRA, A. D. Análise das possíveis mudanças Comerciais e Estruturais do Mercado Internacional de Celulose. 1995. 131 f. Tese (Doutorado em Ciência Florestal) - Universidade Federal de Viçosa, Viçosa, 1995.
RIBEIRO, I. S. A. Análise do Mercado Internacional de Compensado. 2003. 177f. Dissertação (Mestrado em Ciência Florestal) - Universidade Federal de Lavras, Lavras, 2003.

SILVA, O. M. The international market for frozen concentrated orange juice: prospects for Brazil. 1990. $137 \mathrm{f}$. Thesis (PhD) North Carolina State University, Raleigh,1990.

WACK, P. Scenarios: uncharted waters ahead. Harvard Business Review, v. 63, n. 5, p. 73-89. 1985.

ZUGMAN, I. C. Painel geral sobre a produção de compensados. Silvicultura, v. 15, n. 55, p.12-18. 1994.

R. Árvore, Viçosa-MG, v.29, n.2, p.311-320, 2005 